\section{JUSTICIA Y ECOLOGÍA COMO FRONTERA PARA LAS UNIVERSIDADES JESUITAS ${ }^{1}$}

\author{
Juan Antonio Senent de Frutos \\ Universidad Loyola Andalucía \\ jasenent@uloyola.es
}

\section{JUSTICE AND ECOLOGY AS A FRONTIER IN JESUIT UNIVERSITIES}

Cómo citar este artículo/Citation: Senent de Frutos, J. A. (2016). Justicia y ecología como frontera para las universidades jesuitas. Arbor, 192 (782): a365. doi: http://dx.doi.org/10.3989/ arbor.2016.782n6010

Recibido: 04 noviembre 2014. Aceptado: 25 agosto 2015.

RESUMEN: Las universidades jesuitas, en cuanto instituciones que participan de la misión de la Compañía de Jesús han de dar respuesta desde sus funciones a la superación de las fracturas sociales y ecológicas que proliferan en la sociedad global. Esto implica un esfuerzo continuado de traducción e internalización universitaria de esta misión en todas sus actividades propias. En este artículo trataremos de acercarnos a algunas de las implicaciones que tiene esta misión para la vida de las universidades jesuitas. Plantearemos una visión de conjunto de la misión conforme a las últimas congregaciones generales así como el contexto científico y cultural en el que esta traducción universitaria puede darse. Consideraremos en qué líneas puede aportar la propia tradición cultural y espiritual jesuita al tratamiento de la cuestión social y la cuestión ecológica y qué implicaciones tiene esta misión en cuanto a la docencia y aprendizaje, investigación y proyección social en las universidades jesuitas.

PALABRAS CLAVE: Universidad; jesuitas; justicia social; justicia medioambiental; misión; modernidad; tradición ignaciana.
Copyright: (c) 2016 CSIC. Este es un artículo de acceso abierto distribuido bajo los términos de la licencia Creative Commons Attribution (CC BY) España 3.0.
ABSTRACT: Jesuit universities, in as much as they share the mission of the Society of Jesus, must respond, in the framework of their academic commitment, to the social and ecological fractures that proliferate in our global society. This entails a permanent endeavor for all university activities to internalize the mission of the Society of Jesus. This paper suggests some implications of that mission regarding University life. The mission of the Jesuits is considered in relation to recent General Congregations and in the scientific and cultural context in which this adaptation, or internalization, occurs. We will consider how Jesuit cultural and spiritual traditions can illuminate ecology and social justice issues, and the implications of this mission in terms of the teaching, learning, research, and social outreach of Jesuit universities.

KEYWORDS: University; Jesuits; justice; ecology; mission; modernity; Ignatian tradition. 


\section{INTRODUCCIÓN: PLANTEAMIENTO DEL PROBLEMA}

La Compañía de Jesús, que roza ya casi los cinco siglos de historia, es una orden religiosa orientada a la misión en el mundo. Esta misión originaria de la Compañía en su momento fundacional (1540) fue definida como la "defensa y propagación de la fe y el provecho de las almas en la vida y doctrina cristiana"2. Esta diakonia fidei o servicio a la fe, se concretó en campos de trabajo de índole espiritual, social, educativa e intelectual. Así, a lo largo de su historia, todas sus labores, igualmente las educativas y sus contribuciones intelectuales tanto en el ámbito de las ciencias como de las humanidades se debían orientar en última instancia desde el servicio a la fe cristiana. A partir del Concilio Vaticano II y con el generalato de Pedro Arrupe, quien fue Superior General de la Compañía durante el periodo 1965-1981, se desarrolló un proceso renovación en la orientación de su misión en el mundo. Hito fundamental en este proceso fue en la Compañía la Congregación General 32 (1974-1975) donde se declaró que la misión de los jesuitas, el "servicio de la fe" debía incluir también la "promoción de la justicia" como exigencia ineludible de esta fe. Esta orientación no iba destinada sólo a los que trabajaban en obras sociales, sino que se habría de convertir en el "factor integrador de todos los ministerios" ${ }^{3}$, incluido el desarrollado en las instituciones educativas ${ }^{4}$.

Con ello, en este proceso de renovación, la Compañía por medio de las últimas congregaciones generales no sólo ha ampliado el campo de la misión al incluir la dimensión de justicia, no sólo en sentido social e interpersonal, orientada desde su sentido cristiano, sino que la ha conectado y proyectado hacia la justicia ecológica o medioambiental, entendiéndola como cuidado, trato debido y responsable hacia el medioambiente como creación. Con ello, la Compañía se hace también cargo del desafío ambiental al que se enfrenta hoy la humanidad ya que no se puede separar la crisis social de la crisis ambiental planetaria. Responder al desafío de las formas de pobreza, marginación y exclusión en la sociedad global, exige también enfrentar responsablemente el cuidado de la casa común (Francisco, 2015, no 139). Pero este cuidado no es sólo un deber ético, sino que está alimentado y sostenido por una espiritualidad que posibilita una relación más integral con la creación (Álvarez de los Mozos, 2016, p. 83). Ello ha supuesto una ampliación en la definición de la misión por parte de la Compañía vinculada con sus propias fuentes espirituales.

En este contexto, la superación de las fracturas sociales y ecológicas, de la crisis socio-ambiental, que prolifera en la sociedad global se presenta como un desafío transversal para todos los campos de actividad de la Compañía de Jesús. Por ello, los centros de educación superior y las universidades jesuitas ${ }^{5}$, tienen también que afrontar y tratar de dar una respuesta renovada a esta reorientación del horizonte de la misión de la Compañía que en las últimas décadas se está produciendo. Esto implica un esfuerzo continuado de traducción e internalización universitaria de esta misión en todas sus actividades propias.

En este artículo trataremos de acercarnos a algunas de las implicaciones que tiene esta misión para la vida de las universidades jesuitas. Para ello, plantearemos en primer lugar el contexto científico y cultural dominante en el que las universidades jesuitas tienen que desplegar su propio proceder universitario. En segundo lugar, ofrecemos una visión de conjunto de la misión en la que hay que enmarcar su propia actividad universitaria. A continuación, consideraremos en qué líneas puede aportar la propia tradición cultural y espiritual jesuita al tratamiento de la cuestión social y la cuestión ecológica y finalmente qué implicaciones tiene esta misión en la docencia y aprendizaje, investigación y proyección social en las universidades jesuitas. No podemos aquí hacer un tratamiento exhaustivo de las cuestiones implicadas, sino presentar un marco de intelección y de posibilidades que pueden después ser fundamentadas o desarrolladas en posteriores estudios.

\section{CONTEXTO CIENTÍFICO Y CULTURAL PARA LA RENO- VACIÓN DE LA MISIÓN UNIVERSITARIA JESUITA}

Consideremos ahora en sus líneas principales el contexto científico y cultural que ha orientado, y sigue orientando, la misión de la universidad en la Modernidad. No negamos la pluralidad de desarrollos, sino que consideramos su modelo finalmente prevalente en la sociedad moderna, podríamos decir, su "tipo ideal". Este se centra en la producción de un conocimiento social y naturalmente aplicable y en la educación superior de las élites profesionales de esa sociedad ${ }^{6}$. La promoción de la justicia, no suele ser objeto preferente ni propio de la acción universitaria, que se centra fundamentalmente la formación de una sociedad ilustrada y desmitologizada. Por tanto, la única liberación que puede ofrecer la universidad en la Modernidad se centraría en el acceso al conocimiento objetivo que nos procuran las diversas ciencias empíricas que se cultivan en la universidad. Muchos consideran, de palabra o de hecho, que no es misión universitaria contribuir a una transformación social ni a una orien- 
tación racional de la ética individual ni social. Ello se considera un campo exclusivo de las ideologías y cosmovisiones particulares de los diversos miembros, y por tanto de una lucha inacabable e indiscernible entre valores, dada la ausencia de objetividad o la pura subjetividad de los valores y fines humanos, como suele plantearse en este campo desde la hegemónica mentalidad escéptica en cuestiones no empíricas. Esta mentalidad positivista, desplaza también del canon científico a los conocimientos reflexivos. Por ello, tampoco debía ofrecer un autocuestionamiento del canon asumido de racionalidad, una radicalización crítica de los límites de un conocimiento objetivo desvinculado del mundo en el que opera, para ofrecer otros modos racionales de estar en la realidad.

Max Weber, quien expresa y asume rigurosamente el modelo racional de este proceso cultural, nos dirá que el sujeto se orienta por "juicios de valor"; que son de una validez subjetiva, desde un "para mí" de carácter solipsista, no abierto a una validez pública, es decir, no objetivable ni discernible. El conocimiento riguroso y crítico, "universitario", no puede dar cuenta de la asunción de un valor ${ }^{7}$. Lo que puede aportar es un conocimiento objetivo y técnico de los medios para alcanzar los valores asumidos por cada cual, o por la política privada, social o pública. La ciencia, y la universidad con ella, lo que suministrarían es una clarificación sobre la realización fáctica de cualquier finalidad, viendo esta finalidad como a-científica, como algo en cuya racionalidad no puede entrar. En este sentido, la supuesta "racionalidad" del fin o valor perseguido le viene dada desde fuera y se declara "agnóstico" de los valores o fines elegidos. Por ello, los valores e intereses particulares o sociales a los que puede servir la praxis científica y universitaria son sobrepuestos a la misma, son externos, porque estos tienen un carácter o entidad no discernible racionalmente. Por ello, la ciencia se mueve con un método racional en la aprehensión de objetividades, pero en un mundo humano que tiene un carácter finalmente a-rracional. De ahí la radical desorientación, de la que para Weber, no puede escapar el campo científico y universitario, con lo que el mundo humano y no humano, deviene finalmente sin-sentido y desfundamentado. Desde esta situación espiritual en la que se encuentra el científico, y con él el universitario, es posible entender la afirmación de Ortega y Gasset (1957, p. 337), de que "el científico viene a ser el monje moderno". En rigor, para ellos y el mundo moderno no cabría serlo ya de otro modo, aun cuando ello no permita por sí mismo superar el nihilismo de lo humano, como sabe Weber, pues la "tensión entre la esfera de los valores "científi- $\cos ^{\prime \prime}$ y la de la salvación religiosa es totalmente insoluble" (Weber, 2003, p. 230), pero igualmente tampoco la "salvación" o liberación mundana y social es misión propia de la universidad.

Por tanto, en esta concepción prevalente en la Modernidad, ni la universidad ni el progreso del conocimiento, puede tener una misión ética sustantiva desde su misma radicalidad racional. Para Weber, y creo que da cuenta certeramente del límite donde juega la racionalidad de la universidad moderna, o se hace ciencia y transmisión, o se hace política social (Terrén, 1996, p. 133); pero si se hace ésta, entonces ya no se cultiva la ciencia sino la política. Si los científicos sirven o siguen unos u otros valores o fines sería una cuestión de suyo privada, en el sentido en que estaría más allá de su competencia y proceder científico en sentido estricto. Así pues, "la característica del conocimiento científico ha de buscarse en la validez 'objetiva' de sus resultados como verdad" (Weber, 2009, p. 66). Pero esta verdad, sigue enmarcada en una cierta búsqueda de la adecuación entre el conocimiento y la cosa. Por tanto, tiene un carácter estático al pretender inscribirse en el mero plano de la facticidad separado de todo elemento normativo o valorativo de la praxis científica más allá de mera ética interna de la lógica científica o la defensa de ciertos valores epistémicos internos, esto es, de un proceder científico que no se integra en una perspectiva ética mayor con las otras dimensiones de la vida humana, del propio sujeto o del contexto social y material donde se despliega su acción. En esta línea, esta verdad científica se mueve desde el supuesto de la desvinculación del sujeto con el objeto del conocimiento, incluso de otras dimensiones de su propia vida, por eso tanto el científico como el universitario, pueden declararse finalmente irresponsables de la suerte de los otros y de la naturaleza, o de su propia actividad científica. De ahí que esta posición reductora no permita integrar ni ser enriquecida ni por la dimensión ética ni por la dimensión espiritual que podrían tanto articularse desde la propia praxis científica, como entrar en diálogo con ella.

En este contexto, como señaló Benedicto XVI (2011), en medio de la abundancia de conocimientos científicos el conocimiento de lo justo y también nuestra relación con la naturaleza "se ha hecho todavía más difícil". Por un lado, la idea de naturaleza, y por tanto el trato con lo natural, se ha reducido a su sentido funcional, privándola de su dimensión axiológica y ética. Desde ahí, no es posible una lectura normativa de nuestra relación con la naturaleza que habitamos ni con la naturaleza que somos en tanto 
que sujetos vivos. Paralelamente, nuestro ejercicio de la razón se ha reducido para realizar una comprensión puramente científica del mundo, y por tanto, capaz sólo de ofrecernos una imagen del mundo a través de los datos que nos proporcionan las ciencias, pero incapaz de alcanzar el conocimiento del "deber ser", de las cuestiones de valor que nos permitan orientarnos normativamente y por tanto discernir entre lo valioso y lo rechazable. Este contexto cultural, y en cuanto se reduzca la cultura al ámbito de lo científico y expulse de ella a todas las demás convicciones, valores, formas de experiencia y saberes, nos sitúa en una condición de "falta de cultura" normativa o del ámbito del valor (Benedicto XVI, 2011).

¿Cuál es entonces el problema radical de nuestra falta de cultura normativa, por qué se ha llegado a esta agnosia cultural? Es la mirada exclusivamente positivista y funcional ante lo real. Esta posición antropológica hace del ser humano un ser para conocer, utilizar y manipular el mundo y sus estructuras, inclusive, las estructuras que conforman su propia realidad. Como señala el Papa Francisco, se trata de un "antropocentrismo desviado" (2015) ${ }^{8}$. Desde ahí, el ser humano no es capaz de percibir nada más que aquello que es funcional. Y por ello, está cerrado a las otras dimensiones de la realidad. Ello tiene no sólo repercusiones sociales directas, en cuanto puede legitimar la exclusión de las vidas humanas y poblaciones "inútiles", descartables o supuestamente irrelevantes para la marcha de la humanidad, sino también medioambientales. Esta actitud de fondo implica una soberbia utilitaria desde la que no hay límites para el uso o transformación de la naturaleza y donde también se anula el tiempo del proceso evolutivo. Con ello, se desprecia todo vestigio de lo originario de cada especie, del hábitat en el que se sostiene y se da una ruptura con el tiempo de los procesos naturales. A su vez, la explotación intensiva y extensiva de todo el medio ambiente planetario no se detiene ante el ritmo propio de reproducción de la naturaleza, no hace sostenible su huella ecológica, ni reacciona eficazmente con la amenaza del cambio climático global, ni ante los daños sociales que provoca.

Si la acción universitaria quiere hacerse cargo de las exigencias normativas de la realidad humana y no humana, de la justicia social y de justicia medioambiental, tiene que reconstruir el puente que el desarrollo cultural y científico ha roto. ¿Cómo? Tanto la tecnología como los conocimientos científicos tienen que integrarse en un marco mayor y en una nueva sensibilidad. Es necesario "volver a abrir las ventanas, hemos de ver nuevamente la inmensidad del mundo, el cielo y la tierra, y aprender a usar todo esto de modo justo" (Benedicto XVI, 2011). Ampliar nuestro ejercicio de la razón exige ampliar nuestros registros en el acceso y en el trato hacia los seres naturales y para ello es preciso modificar nuestra actitud fundamental ante lo real; acceder a una nueva sensibilidad, un "corazón dócil" hacia las enseñanzas de la naturaleza.

Para esta recuperación, se necesita un diálogo entre conocimientos científicos, saberes y tradiciones culturales y religiosas diversas. Precisa de una ecología de saberes polifónica en cuanto pueden enriquecerse y sumar esfuerzos en el cuidado de nuestro mundo. Las diversas posiciones seculares y religiosas (que juegan en el espacio de luchas de la cultura moderna a veces como excluyentes ${ }^{9}$, pueden también complementar$\mathrm{se}^{10}$ ), como también contribuyen las culturas minorizadas en la exterioridad moderna como los pueblos indígenas y las sociedades tradicionales.

En este contexto, converge la capacidad civilizatoria también de la espiritualidad cristiana en la tarea de promover las exigencias de justicia ante el conjunto de la creación, ante los seres humanos, ante los otros seres vivos, y también ante la propia naturaleza humana en riesgo de manipulación. Por ello, la tradición cristiana tiene hoy una función tanto a nivel de la ecología política como de la "ecología humana", ante el cuidado y respeto de las vidas, de la biodiversidad y de la identidad humana ante el futuro. Es la tarea de promover una "ecología integral" (Camacho Laraña, 2016, pp. 69-70; López Casquete de Prado, 2016). La lectura creyente ante la realidad que reconoce en ella a un Dios creador y sostenedor de mundo, que nos convoca a reconciliarnos entre los seres humanos y con la creación; a un particular "reencantamiento del mundo" que supera normativamente el moderno "desencantamiento del mundo", ya que conduce a relacionarnos con el mundo que somos y habitamos desde el deber de reconocimiento, respeto y cuidado. De este modo, se da un espacio de convergencia desde la tradición cristiana, y en ella la tradición ignaciana y jesuita, junto con otras religiosas y seculares, que permite otro acceso normativo ante lo real y puede orientar de otro modo la actividad de la universidad.

En suma, si contrastamos la misión universitaria desde la idea hegemónica en la modernidad con la misión que buscan la Compañía y sus universidades tendremos que señalar que la incorporación de la promoción de la justicia y la ecología en la misión universitaria tiene, como veremos a continuación, un carácter contracultural con respecto al modelo universitario dominante. Por tanto, implica orien- 
tarse hacia otro modelo alternativo de acción universitaria y precisamente por ello, puede tener una pertinencia mayor para enfrentar los retos socioambientales de la sociedad global actual. En este sentido, la perspectiva de la Compañía puede también participar o confluir con otras visiones y esfuerzos contemporáneos por realizar la misión universitaria en otra dirección no hegemónica.

\section{LA MISIÓN DE LA COMPAÑÍA DE JESÚS COMO HORI- ZONTE DE SUS UNIVERSIDADES}

En este apartado, al tratar de la misión de la Compañía de Jesús, mostraremos el horizonte en el que se enmarca y debe desenvolverse la vida de las universidades jesuitas. En la solicitud porque todas las obras de la Compañía y en particular sus universidades se orienten desde sus especificidades hacia la misión común, hay que recordar el exigente llamamiento que la Congregación General 34 realiza en su decreto sobre la Compañía y la vida universitaria: "Para poder llamarse institución [universitaria] de la Compañía, habrá de evaluar y responder periódicamente ante ésta a fin de comprobar si su dinámica se desarrolla o no en línea con la misión de la Compañía. Los que trabajan en tales Universidades, en cuanto comunidad y en cuanto personas concretas, deberán dedicarse activamente a la institución, orientándola a los fines que la Compañía aspira a lograr de ella" (Congregación General 34, Decreto 17, no 9).

Así pues, este será el marco de sentido donde están llamadas a inspirarse estas universidades y a orientar sus actividades específicas, y desde donde hay que entender hoy la promoción de la justicia socioambiental, esto es, la atención a la cuestión social y a la cuestión ecológica, como elemento transversal a cultivar en la acción universitaria jesuita.

Como hemos señalado, la misión originaria de la Compañía en su fundación fue identificada como la "defensa y propagación de la fe". En la postconciliar Congregación General 32 se reformuló esta misión como "defensa de la fe y promoción de la justicia"11. Esta novedad, supuso no sólo un cambio de orientación mental y de lenguaje, sino más bien una cierta metanoia, una conversión del corazón y de la sensibilidad, que incluía la búsqueda de una nueva coherencia de vida, de estilo y de relaciones. Como el propio superior general Peter Hans Kolvenbach reconoció en el año 2000, a los veinticinco años de aquella reorientación, no se trató de un cambio fácil, sino de un camino de cruz, de resistencias, oposiciones eclesiales y externas, pero que finalmente se terminó confirmando como la misión que identificaba pública e internamente a la Compañía de Jesús (Kolvenbach, 2000/2008, p. 174).

El servicio de la fe, según la Congregación General 32 , se debía entender en esta nueva fase purificada de elemento de proselitismo y de imposición. Se trataba de presentar a los otros el mensaje de Jesús, no de un modo reactivo y combativo, sino más bien como una proclamación. Según Kolvenbach (2000/2008, p. 175) "en el contexto de siglos de espiritualidad jesuita, el servicio de la fe no puede significar otra cosa que llevar a nuestro mundo el don contracultural de Cristo". Un trabajo por la fe de Cristo, que se quiere llevar a cabo según el mismo estilo de servicio de Cristo. Se trata de proseguir el proyecto de Jesús de gastar la vida para la salvación de todos, en un mundo donde está muy presente y activo el sufrimiento, la marginación y la exclusión de muchos. Por ello, en la profundidad de esta diakonia fidei, ya estaba implicada la dimensión de promoción de la justicia.

¿Cuál es la idea de justicia que opera en el uso de la Compañía de Jesús? Como se ha señalado, "no puede encontrarse en las Congregaciones Generales una definición normativa de la justicia" (Secretariado para la Justicia Social y la Ecología, 2014, p. 12). Sin embargo, hay un entendimiento común que puede verse reflejado en el uso de la idea de justicia en los documentos autoritativos de la Compañía en las últimas décadas. Se trata de una aproximación que se mueve más en un horizonte semítico ${ }^{12}$ que helénico. No se trata primero de conocer el principio formal de lo justo para después caminar hacia la realización de un canon, de un ideal de perfección como en la tradición griega. Se mueve en una aproximación más inductiva que deductiva. Y por tanto, lo primero que se indaga y se atiende es a lo real tal como se nos manifiesta en nuestra experiencia. Esta experiencia es leída desde una sensibilidad, guiada por una fe que reconoce en lo humano la presencia e interpelación de lo divino. El centro del valor está en las vidas humanas concretas, no en las normas o ideales que deben regir la vida humana. Así, el centro de gravedad no es un criterio racional de ordenación de la vida sino que el centro de valor son los seres humanos, seres que se viven como necesitados, tensados hacia su desarrollo o plenificación, relacionales o abiertos y codependientes de los otros y muchas veces imposibilitados por estructuras sociales que les sitúan en un mal vivir. La justicia, será así la superación de la injusticia ${ }^{13}$, pues no se opera desde un principio lógico sino experiencial. Lo que sea la justicia, lo iremos descubriendo en la medida 
en que nos enfrentamos a las injusticias. Lo que encontramos es un mundo en el que está presente para muchos la pobreza, el sufrimiento, la no libertad y la insolidaridad de los que viven bien a costa de la explotación de otros o de la indiferencia ante la suerte de los más débiles; un mundo que clama por su superación. El proceso práctico-real de superación de estas situaciones de negatividad humana y social dará lugar a la formulación y reivindicación de valores que guíen el proceso, de reglas racionales que alienten la superación de un orden irracional en cuanto anule o impida la vida. Pero ello sería un momento segundo y derivado. En este sentido, podemos entender que la fórmula que en la Compañía se suele emplear para expresar el servicio en favor de la justicia es el de "promoción de la justicia"14. Esto da cuenta de que se parte de una instancia concreta y real, pues la promoción implica elevación o mejora de las condiciones de vida (tercera acepción según el diccionario de la RAE). Por tanto, no tiene en primer término un sentido normativo, pues no se trata de "cumplimiento". Este trabajo de promoción se entiende asimismo, como una tarea de "reconciliación", por tanto de establecimiento de una relación benéfica entre los seres humanos divididos, de acercamiento y recomposición de las relaciones antagónicas para que sea posible un nuevo vivir bien en solidaridad de unos con otros. Por ello, esta dimensión social de la justicia, está animada a su vez por la "justicia del Evangelio", en línea con el amor y la misericordia presentes en el mensaje del Reino de Dios, que reconstruye las fracturas en las relaciones.

En suma, la justicia es entendida como relación benefactora y correcta entre los sujetos. Es por tanto, en primer lugar una relación viva y que posibilita la vida buena. Se trata de una instancia material, que podrá ser orientada conforme a normas y valores, entendidos como mediaciones al servicio de la realización de la justa relación que posibilita de la vida plena de los sujetos.

Dos décadas después, en 1995, la Congregación General 34 contextualizó y resignificó aún más esta misión: "nuestra misión de servicio a la fe y promoción de la justicia debe ensancharse para incluir dimensiones esenciales para la proclamación del Evangelio, el diálogo, y la evangelización de la cultura; hemos insistido en la inseparabilidad de la justicia, el diálogo y la evangelización de la cultura" (Congregación General 34, Decreto 4, no. 6.). ¿Por qué esta conexión fe-justicia-diálogo-cultura? La misión no puede realizarse sino desde el diálogo a partir de los diversos contextos con sus tradiciones culturales y religiosas y desde la cooperación con otros, como propuso el Secretariado para la Justicia Social (2006, p. 79). En este contexto de misión, señalaba Adolfo Nicolás a propósito del apostado intelectual que este "traza un camino de diálogo entre el Evangelio y las culturas, las ciencias y las tradiciones religiosas" (Carta Sobre los jesuitas destinados al apostolado intelectual). Puede haber momentos de encuentro, de enriquecimiento mutuo y también de crítica y de trabajo común con otros en cuestiones en las que está en juego la dignidad humana.

Este proceso de reorientación de la misión en el mundo de la Compañía de Jesús que comenzó formalmente en 1975, tiene su actualización en 2008 con la Congregación General 35 donde se define la autocomprensión actual del sentido de la presencia en el mundo de este cuerpo social y de sus obras. Lo más significativo de esta congregación general es que se confirman los campos de la misión definidos desde la Congregación General 32, integrándolos en una comprensión unitaria y no meramente aditiva ${ }^{15}$, y a su vez, que se amplía el campo de la promoción de la justicia con el cuidado de la creación, la justicia social con la justicia medioambiental. Signo expresivo de la ampliación de este campo es que el órgano que desde gobierno universal de la Compañía trata de animar y coordinar la promoción de la justicia, el Secretariado para la Justicia Social, pasó a nombrarse desde entonces, Secretariado para la Justicia Social y la Ecología (SJSE) ${ }^{16}$.

Sin embargo, aunque supuso una confirmación, la inclusión de la perspectiva ecológica en el núcleo de la misión de la Compañía estaba ya haciéndose presente en las últimas décadas. En 1983, la Congregación General 33 se reconoce el problema ecológico y su interdependencia con el modo de relacionarse con Dios y entre los hombres: "Al despreciar los hombres el conocimiento del Amor Creador rechazan también la dignidad de la persona humana y destruyen la misma naturaleza creada" (Congregación General 33, Decreto $1, \mathrm{n}$-. 35). Este fue el inicio visible del surgimiento de la conciencia ecológica en los centros sociales de la Compañía (Secretariado para la Justicia Social y la Ecología, 2013, p. 41). En la siguiente década, para la Congregación General 34 se presentaron dos grupos de postulados ${ }^{17}$ sobre esta cuestión. En el primer grupo, se subrayaba la mutua conexión entre la promoción de la justicia y el desafío planteado por la degradación del medio ambiente, la interconexión sociedad-economía-ecología, y en especial con relación a los más pobres. La "opción por los pobres" se consideraba en 
íntima conexión con la "opción por la tierra", pues la suerte de los pobres va de la mano de la suerte de la tierra. En el segundo grupo de postulados, se mostraba la coherencia que existe entre la espiritualidad ignaciana y la sensibilidad por el cuidado de la creación. Esta dimensión ecológica debía, en consecuencia, afectar al estilo de vida propio y al modo de proceder de la Compañía. Por ello, debería estimularse la reflexión teológica y la investigación sobre problemas ecológicos y promover la integración de la perspectiva ecológica en la formación y en todos los ministerios de la Compañía (Miralles, 1995, pp. 363-364). La Congregación General 34 no pudo elaborar un decreto sobre ecología, y dejó una recomendación al padre general para realizar un estudio que fuera comunicado a toda la Compañía. Los resultados de este estudio, se publicaron en el documento "Vivimos en un mundo roto: reflexiones sobre ecología"18.

En esta línea prosigue la Congregación General 35 confirmando esta misión de servir la fe, promover la justicia social y ecológica, y el diálogo con otras culturas y religiones. Si este es el campo de misión, el lugar particular es en las fronteras, así lo marca el Decreto 3 de la misma en su propio título: "Desafíos para nuestra misión hoy. Enviados a las fronteras". Las fronteras son ámbitos no bien conocidos, complejos, con reglas de juego aún no aprobadas, que pueden llevar a conflictos (Guibert, 2014, p. 220) y en los que se juega las transformación social y cultural. ¿Qué fronteras? Las fronteras sociales y culturales que se alzan entre el evangelio y la vida de la humanidad hoy (Congregación General 35, D3, 19-24), las que impiden a las mayorías del planeta una vida digna en plenitud (Congregación General 35, Decreto 3, pp. 25-30) y amenazan la propia vida del medio ambiente (Congregación General 35, Decreto 3, pp. 31-36). Fronteras que deben ser removidas desde el esfuerzo común con otros (Congregación General 35, Decreto 6) y en diálogo con sus respectivas tradiciones culturales y religiosas. Para superar las carencias actuales de la sociedad global, para corregir sus fracturas, no basta con el necesario recurso a la ciencia y a la tecnología, sino que es preciso también un diálogo con las tradiciones culturales y religiosas que pueden tener una palabra significativa en la reorientación de nuestro futuro como humanidad.

Por ello, en este contexto, la Congregación General 35 expresa también un para qué de la misión de la Compañía, que se entiende como la tarea de establecimiento de relaciones justas en clave de reconciliación de las tres grandes fronteras que fracturan el mundo, en lo que se ha denominado un tríptico de relaciones (Secretariado para la Justicia Social y la Ecología, 2011, p. 32): “En este mundo global, marcado por tan profundos cambios, queremos profundizar ahora nuestra comprensión de la llamada a servir a la fe, promover la justicia y dialogar con la cultura y otras religiones a la luz del mandato apostólico de establecer relaciones justas con Dios, con los demás, y con la creación" (Congregación General 35, Decreto 3, p. 12). En primer lugar, reconciliación con Dios, en el contexto de la problemática relación de los hombres y mujeres con Dios desde la cultura dominante secular, individualista, materialista y postmoderna. En segundo lugar, reconciliación de unos con otros en una sociedad global desigual, con dinámicas neocoloniales, para tender puentes entre pobres y ricos. Así, el trabajo intelectual que se realiza desde la Compañía, "nos proporciona una ayuda inestimable para establecer estos puentes, ofreciéndonos nuevos modos de entender en profundidad los diversos mecanismos e interconexiones de los problemas actuales" (Congregación General 35, Decreto 3, p. 28). Conocimiento en profundidad de los mecanismos de las injusticias sociales que permite, a su vez, ofrecer nuevas posibilidades de desarrollo social. Y en tercer lugar, reconciliación con la creación. El cuidado del medioambiente, está interrelacionado con las otras fronteras, pues "afecta a la calidad de nuestra relación con Dios, con los otros seres humanos y con la misma creación" (Congregación General 35, Decreto 3, p. 31). El modo actual de explotar las fuentes energéticas y otros recursos naturales está dañando el equilibrio ecológico y amenazando el futuro en el planeta. Quienes más están sufriendo las consecuencias medioambientales de los diversos deterioros son los pobres. Comunidades pobres han sido desplazadas, al igual que pueblos indígenas (Congregación General 35, Decreto 3, p. 33). En este contexto, esta Congregación urge a todos los jesuitas y a quienes comparten la misma misión, en particular a las universidades y centros de investigación, a promover estudios y prácticas orientadas a enfrentar las causas de la pobreza y a mejorar el medio ambiente: "Debemos encontrar caminos en los cuales nuestra experiencia con los refugiados y los desplazados, por una parte, y con las personas que trabajan en la protección del medio ambiente, por otra, interactúen con aquellas instituciones, de forma tal que los resultados de la investigación y la incidencia política consigan beneficios prácticos para la sociedad y el medio ambiente. Esta incidencia política $e$ investigación deberían estar al servicio de los pobres 
y de quienes trabajan en la protección medioambiental ${ }^{19 "}$ (Congregación General 35, Decreto 3, p. 35).

Con ello, la formulación de la misión en la Congregación General 35 recoge, pues, un proceso de ampliación y de explicitación del campo con respecto a las últimas décadas e incluso de su expresión formal originaria, pero un mismo pathos de servicio transformador en el mundo. El lugar de destino, no es ya un simple lugar físico, es ubicuo, se encuentra "en todas partes", quizá porque la "redondez" de la tierra lo ha hecho más interconectado, interdependiente y circular que nunca por dinámicas de interacción, que también están atravesadas por la violencia y el sufrimiento ${ }^{20}$ y el deterioro medioambiental. Por ello, si es pertinente la promoción de la justicia como parte inseparable de la misión de servicio al Evangelio, la justicia animada desde la fe cristiana, abre también una serie de posibilidades que pueden ser realizadas desde las universidades jesuitas. Así, podemos entender también que la promoción de la justicia exige el trato respetuoso a las culturas y entre las culturas y sus creencias y religiones (justicia intercultural e interreligiosa); y parte de esta justicia intercultural es el justo diálogo entre ciencias, culturas y tradiciones religiosas (justicia epistémica). A su vez, una fe y espiritualidad que nos conecta y sitúa en toda la realidad natural del mundo, nos demanda hoy una justicia ecológica.

El último hito a destacar en la orientación de esta misión, ha sido la celebración de la última Congregación General de la Compañía de Jesús (36) entre octubre y noviembre de 2016, donde además de elegir un nuevo Superior General, P. Arturo Sosa, se ha deliberado en dos líneas fundamentales. La primera, el gobierno de la Compañía y sus estructuras; y la segunda, sobre la vinculación entre la "vida y misión de la Compañía"21. Sobre esta segunda línea, señala Velasco (2016) "nuestras preguntas deben ir más dirigidas al modo como vivimos lo que hacemos, y de qué modo ese hacer es testimonio del Reino de Dios que anunciamos (...) De qué maneras nuestras opciones comunitarias en cuanto a los lugares donde vivimos, en favor de quienes estamos, cómo nuestras comunidades están abiertas a los más pobres, cercanas a las necesidades de los sufrientes, son signos de nuestra misión". La interpelación que de esto surge para la misión a encarnar universitariamente es evidente. Cabría preguntarse de qué modo la vida y las opciones de las universidades en sus tareas propias son manifestaciones que actualizan y reflejan la misión que están llamadas a encarnar. Ello implica, recogiendo el dicho del Papa Francisco en su diálogo con los jesui- tas congregados (Diálogo del Papa Francisco con la 36a Congregación General de la Compañía de Jesús), "tener coraje y audacia profética" para no quedarse en resistencias o miedos ante la misión asumida, sino discernir y desarrollar las opciones y modos universitarios que más actualicen y testimonien el servicio humanizador en el mundo de hoy.

\section{EL APORTE A LA JUSTICIA Y LA ECOLOGÍA DESDE LA TRADICIÓN ESPIRITUAL Y CULTURAL JESUITA}

La concepción prevalente de la vida científica y universitaria expresa la traducción universitaria de una línea hegemónica de desarrollo cultural de la Modernidad. De aquí podemos decir que, según sea el marco cultural en el que se sitúe la actividad universitaria, su orientación será diversa. Por ello, si un quehacer universitario se encarna vitalmente en una tradición cultural particular ${ }^{22}$, podrá liberar posibilidades distintas de desarrollo intelectual y social. De ahí que la apropiación crítica y reflexiva de una tradición permita no sólo reorientar su misión sino su propio modo de proceder universitario. Si esta tarea se realiza reflexivamente, tendrá que aplicar un "espíritu de discernimiento". Se trata con ello, de elegir entre los modos de hacer ciencia y de educar, no sólo que tengan sentido y familiaridad para sus actores, sino aquellos que mejor sirvan para responder a las necesidades de nuestro mundo.

El Decreto sobre la Compañía y la vida universitaria de la Congregación General 34 señalaba que la actividad universitaria jesuita, ha de entenderse como la unión de un sustantivo, universidad, y un adjetivo, jesuita, y que hay que estar muy alerta para que el sustantivo y el adjetivo, "sean siempre respetados" (Cf. Congregación General 34, Decreto 17, n 5). La constante histórica de la institución universitaria en Occidente desde su origen medieval hasta nuestros días es de una institución que se vertebra sobre el cultivo del conocimiento para la búsqueda y transmisión de la verdad. Si vemos en perspectiva histórica esta institución, enseguida resalta que la concreción del modo de entender el conocimiento, las ciencias y su relación con la sociedad y su destino cambia según el marco cultural en el que se sitúa. En realidad, nunca es posible el cultivo del conocimiento sin un horizonte cultural particular, lo cual no obsta a que pueda haber cierta circularidad entre la búsqueda de la verdad y el modo en que la verdad es vista en un contexto sociocultural, en el sentido de una lenta y afanosa transformación del horizonte de intelección desde el propio proceso del conocimiento. Dicho de otra ma- 
nera, toda vida universitaria está siempre adjetivada, se declare o no. Otra cosa es que haya que tener gran cuidado en que el lugar cultural y social desde el que se hace universidad no sea simplemente el que se promueve desde los poderes sociales hegemónicos y sea el más inclusivo y universalizable, el debido y el que se dice querer seguir.

En este sentido, la Compañía es consciente de que su ya multisecular tradición universitaria no se ejerce simplemente orientada por una misión, sino desde una "visión", desde un modo de proceder que está alimentado por una tradición cultural ${ }^{23}$ que se inspira en una particular espiritualidad cristiana, la espiritualidad ignaciana, que alimenta la misión de la Compañía y el modo de realizar esta misión y orienta también su visión de la misma. No se trata de que esta espiritualidad provea de modo automático o mecánico este desempeño, sino de que el quehacer de los que trabajan en la universidad se ayude activamente desde los medios que provee esta espiritualidad para los sujetos que se quieren servir de ella. En este contexto, como ha señalado el Secretariado para la Justicia Social y la Ecología, "las universidades de la Compañía tratan de promover la justicia en el medio universitario dentro de la tradición ignaciana" (Secretariado para la Justicia Social y la Ecología, 2014, p. 12). Si hoy la promoción de la justicia y la ecología deben forman parte de la misión de la Compañía y de sus universidades, esta misión puede ser inspirada a su vez en su propia tradición espiritual en diálogo con otras tradiciones. Desde que la Congregación General 32 estableció en el centro de la misión de la Compañía el binomio "servicio de la fe y promoción de la justicia", se ha tratado de contextualizar y proyectar esta misión desde las fuentes espirituales de la misma ${ }^{24}$. Esta espiritualidad provee de una sensibilidad y de un horizonte de intelección a la actividad intelectual desplegada en las universidades desde la centralidad del pobre y el reverso de la historia. Conforme a la tradición teológica cristiana, Dios se ha vaciado de sí y se ha encarnado en Jesús, pobre y humilde: "Las fronteras de la pobreza, la marginación, la injusticia, la inhumanidad, son espacios privilegiados donde encontrarse con Dios y profundizar en el misterio de la realidad. Mirar la realidad desde abajo, desde los pobres, desde sus sufrimientos, luchas y esperanzas es un modo preferible de acceso a la verdad" (SJSE, 2014, 13). Desde aquí se constituye esta visión.

En esta línea de inspiración de la misión en la propia tradición, la Congregación General 34, en la recomendación al padre general sobre ecología, se subraya la coherencia que existe entre la espiritualidad de los Ejercicios y la sensibilidad por la integridad y el cuidado de la creación. Por ello recomienda realizar un estudio sobre "cómo nuestra espiritualidad ignaciana nos proporciona base para una respuesta universal [sobre ecología]" (Congregación General 34, Decreto 20, no. 2). En este contexto, en las dos últimas décadas, están aflorando estudios ${ }^{25}$ y reflexiones ${ }^{26}$ sobre la tradición ignaciana y la ecología ${ }^{27}$, que muestran cómo la espiritualidad ignaciana tiene mucho que contribuir a la creación de un medio ambiente sostenible, y además, se está ya realizando una integración de la dimensión ecológica en la práctica de la espiritualidad a través de diversos medios (Secretariado para la Justicia Social y la Ecología, 2013, pp. 48-50).

\section{A MODO DE CONCLUSIÓN: JUSTICIA Y ECOLOGÍA COMO FRONTERA PARA LAS UNIVERSIDADES JESUITAS}

La misión para el mundo a la que es enviada la Compañía de Jesús en sus diversas obras por medio de la Iglesia, esto es, servicio de la fe y promoción de la justicia social y la ecología en diálogo con las culturas y tradiciones religiosas del mundo, representa un todo unitario que tiene una coherencia e implicación interna y sistemática de estas cuatro dimensiones (Romero Rodríguez, 2007, pp. 400-403). En aras de hacerla operativa se puede segmentar el enfoque de algunas de estas dimensiones en los respectivos campos de actividades. En estas consideraciones finales, nos enfocamos desde ese contexto a la dimensión de la justicia y la ecología para las universidades jesuitas.

Para expresar el lugar real en que se juega su misión, la Compañía, sobre todo a partir de la Congregación General 35 (Decreto 3), usa la metáfora de la frontera: "Desafíos para la misión hoy. Enviados a las fronteras". Ello da cuenta de que la misión tiene un carácter vital e incluso agónico, de lucha por la vida en contra de aquello que le impide subsistir y plenificarse. En la "frontera" se puede jugar la posibilidad de existencia de una sociedad y los condicionamientos para su subsistencia futura. Donde, como ha señalado Guibert (2014, p. 220), no hay reglas fijas o conocidas, y por tanto hay que estar en permanente alerta y tensión creativa para dar con las estrategias adecuadas y en proceso también de transformación para responder a un contexto cambiante. Recogiendo esta metáfora y su potencialidad significativa, las universidades jesuitas también están convocadas a estar en la frontera para atender, desde sus propias funciones, a la misión universal de la Compañía. La actitud fundamental para ello que la Compañía pide a sus universidades 
es la reflexividad permanente: "En cuanto [universidades] jesuitas, buscamos el conocimiento por sí mismo, pero debemos preguntarnos de continuo sobre el para qué del conocimiento" (Congregación General 34, Decreto 17, no. 6). Por tanto, aquí la universidad, no es meramente el lugar del cultivo del saber y de su transmisión, sino que está dinamizada también por la pregunta del para qué que debe proyectar en la funciones universitarias propias como la investigación, la docencia y aprendizaje, y la proyección social.

En este contexto, de "estar enviados a las fronteras", la Compañía se encuentra en un proceso de renovación interna en las últimas décadas. Las universidades de la Compañía, en este proceso, han podido a veces situarse al margen de esta renovación dadas sus características diferenciales (Eguiluz, 1995, p. 340). Quizá por eso, la Congregación General 34, el decreto "la Compañía y la vida universitaria", lo organiza en torno a dos grandes retos, "El desafío de las estructuras de las Universidades", y "Fe y justicia como desafío a las Universidades", es decir, el desafío de las estructuras y el de la misión. Por ello, reconociendo las adaptaciones (Congregación General 34, Decreto 17, no. 8) que muchas universidades han hecho inspiradas por el Decreto 4 de la Congregación General 32, invita a encarnar en su "contextura institucional" (Cf. Congregación General 34, Decreto 17, n. 7) esa misión. Se trata de estar despiertos para que la institución se oriente desde la misión y no desde sus propias dinámicas e intereses autorreferenciales: "pensando en el futuro tenemos que estar muy alerta para que el sustantivo "universidad" y el adjetivo "jesuítica" sean siempre plenamente respetados" (Cf. Congregación General 34, Decreto 17, n. 5). En el contexto de los desafíos de la propia estructura institucional, recordamos aquí, la petición del entonces Superior General de la Compañía (2008-2016), Adolfo Nicolás, que es especialmente pertinente para enfrentar los desafíos globales en el campo de la justicia social y medioambiental. Nicolás pedía a las universidades jesuitas "re-descubrir" la universalidad, para formar una "coherente red universitaria jesuita (...), ¿no podríamos hacer mucho más si ampliamos el objetivo de nuestro servicio al mundo, si todas las instituciones de Educación Superior Jesuita forman, un proyecto social único global?" (Profundidad, universalidad y ministerio intelectual, p. 9), al servicio de un "mundo humano, justo y sostenible" (Profundidad, universalidad y ministerio intelectual, p. 1). Esta red, o redes que progresivamente se deben ir articulando, se deberían centrar en problemas supranacionales importantes: "dignidad de la vida humana, la promoción de la justicia para todos, la calidad de la vida personal y familiar, la protección de la naturaleza, la búsqueda de la paz y estabilidad política, un reparto más justo de los recursos mundiales, y un nuevo orden económico y político, que sirva mejor a la comunidad humana a nivel nacional e internacional" (Profundidad, universalidad y ministerio intelectual, p. 9). Es evidente, que una estructuración en red de las universidades jesuitas, y enfocadas al tratamiento de estas cuestiones vitales para el presente y el futuro de la humanidad permitiría un mejor servicio social y ambiental desde sus propias funciones ${ }^{28}$.

En coherencia con lo anterior, cabe preguntarse cómo se pueden caracterizar y dinamizar las funciones de una universidad jesuita. La respuesta se mueve en la búsqueda de una "excelencia universitaria jesuita" (Secretariado para la Justicia Social y la Ecología, 2014 , p. 18). Esta excelencia incluye la excelencia académica a la que aspira cualquier universidad, pero la tensiona buscando la calidad humana y social de sus procesos. Calidad que se orienta por un pathos transformador e integrador de valores, conocimientos y competencias al servicio de educación de la persona y de su realización en el mundo. Es lo que se formula en el denominado paradigma Ledesma-Kolvenbach ${ }^{29}$ como objetivos de la Educación Superior, el cultivo integral de la "utilitas, iustitia, humanitas, fides", según la lectura que del jesuita Ledesma (s. XVI) realizó el Superior General de la Compañía (1983-2008) Peter Hans Kolvenbach (2000/2008). Para Kolvenbach, el criterio real de evaluación de las universidades de la Compañía radica en lo que los estudiantes lleguen a ser $(2008 b, 182)$, es decir, en la calidad humana que alcanza el estudiante, no solo en el campo profesional o intelectual, sino también en el psicológico, moral y espiritual (Kolvenbach, 2006/2008, p. 343). Con ello, se trata de "educar para una ciudadanía responsable en la ciudad del mundo" de formar "líderes preocupados por la sociedad y el mundo, deseosos de acabar con el hambre y los enfrentamientos en el mundo" (2006/2008), diríamos hoy, personas con pasión por la justicia social y medioambiental.

En el campo de la investigación al servicio de la promoción de la justicia social y ecológica en las universidades jesuitas, tanto Kolvenbach (2000/2008, p. 12) como Nicolás (Profundidad, universalidad y ministerio intelectual), han enfatizado el carácter orientado y transformador que deben tener las investigaciones universitarias, al servicio de los grandes problemas humanos, sociales y ambientales de nuestro tiempo, como los antes señalados. En este sentido, también la Congregación General 35, urge a las universidades 
jesuitas a "promover estudios y prácticas orientadas a las causas de la pobreza y a mejorar el medio ambiente" (Decreto 3, no. 35).

Pero a su vez, si los intereses y objetos han de estar orientados, es preciso también una renovación metodológica en las disciplinas científicas que las haga más permeables a colaborar entre sí dado el carácter interdependiente de los fenómenos humanos, sociales y naturales, y la necesidad también de avanzar desde el conocimiento a la sabiduría, esto es, de un conocimiento integrado al servicio del crecimiento o la autorrealización humana. En esta línea, hay reiterados Ilamamientos desde la Congregación General 32 hasta hoy, a favorecer y cultivar el diálogo interdisciplinar como medio de avanzar en la tarea. En esta marcha, y recogiendo nuevas propuestas metodológicas de nuestro tiempo, el filósofo jesuita colombiano Gerardo Remolina, propone avanzar desde el cultivo de las disciplinas y el diálogo interdisciplinar, hacia la "transdisciplinariedad" (Remolina, 2012, p. 9). En este proceso de renovación metodológica al servicio de la misión, ya están embarcadas algunas universidades jesuitas (Secretariado para la Justicia Social y la Ecología, 2014, p. 30).

Finalmente, la excelencia de una universidad jesuita en su proyección social, se cualifica por llegar a constituir una fuerza social, integrando sus funciones y sectores específicos en una totalidad coherente y eficaz, que está llamada "a vivir para [la] realidad social, a iluminarla con la inteligencia universitaria, a emplear todo el peso de la universidad para transformarla. Así pues, las universidades de la Compañía tienen razones más fuertes y distintas a las de otras instituciones académicas o de investigación para dirigirse al mundo actual, tan instalado en la injusticia, y para ayudar a rehacerlo a la luz del Evangelio" (Kolvenbach, 2000/2008, p. 13)

\section{NOTAS}

1 Este trabajo se enmarca en el Proyecto de Investigación Pensamiento y tradición jesuita y su influencia en la Modernidad desde las perspectivas de la Historia, la Traductología y la Filosofía Jurídica, Moral y Política FFI2015-64451-R (MINECO/FEDER), financiado por el Ministerio de Economía y Competitividad y el Fondo Europeo de Desarrollo Regional (FEDER). Investigador Principal: Juan Antonio Senent de Frutos.

2 Fórmula del Instituto de la Compañía de Jesús que fue confirmada en 1550 por la bula papal Exposcit debitum: así, la Compañía es "fundada ante todo para atender principalmente a la defensa $y$ propagación de la fe y al provecho de las almas en la vida y doctrina cristiana por medio de predicaciones públicas, lecciones, y todo otro ministerio de la palabra de Dios, de ejercicios espirituales, y de la educación en el Cristianismo de los niños e ignorantes, y de la consolación espiritual de los fieles cristianos, oyendo sus confesiones, y administrándoles los demás sacramentos. $Y$ también manifiéstese preparado para reconciliar a los desavenidos, socorrer misericordiosamente y servir a los que se encuentran en las cárceles o en los hospitales, y a ejercitar todas las demás obras de caridad, según que parecerá conveniente para la gloria de Dios y el bien común, haciéndolas totalmente gratis, y sin recibir ninguna remuneración por su traba- jo, en nada de lo anteriormente dicho" (Ignacio de Loyola, 1997, p. 455).

Puede también hacerse una lectura de la Fórmula inclusiva de la dimensión de justicia. Guillermo Rodríguez-Izquierdo (2014) destaca que lo que se propone después de las obras de servicio de la fe es "no menos" [nihilominus en la redacción latina oficial, significando aquí "no menos" o "lo mismo"] importante que lo que se ha dicho antes con respecto al servicio de la fe. En esta línea, destaca que lo que se propone tras el "Y también manifiéstese preparado...", con el lenguaje de aquel tiempo, son obras de "promoción de la justicia" para la atención a necesitados o excluidos. Así, el servicio para la "gloria de Dios" no está desconectado sino imbricado con el servicio del "bien común", cuya promoción se orienta inclusivamente desde los necesitados. Por tanto, se trata de una posible y legítima lectura ya presente en la propia Fórmula, que sería confirmada autoritativamente en la Compañía a partir de 1975 al definir la misión de la Compañía en el mundo de hoy.

3 Congregación General 32, Decreto 2, n. 9.

4 Congregación General 32, Decreto 2, n.9 y Decreto 4, n. 76.

5 Entendemos el término "universidades jesuitas" en un sentido amplio, incluyendo tanto a aquellas que son propiedad y están bajo la dirección formal y directa de la Compañía, como aquellas que sien- do entidades jurídicamente independientes tienen una supervisión por parte de la Compañía y se declaran inspiradas en la tradición universitaria jesuita. Unas y otras serían realmente jesuitas cuando sirven a la misión de la Compañía (Cf. Congregación General 34).

6 Así, por ejemplo, Ortega y Gasset en su clásico ensayo La misión de la universi$d a d$, señala que esta consiste en educación técnica superior o especializada e investigación científica; junto a estas dos funciones principales, rescata un "residuo" medieval, la formación en "cultura general". Sin embargo, en la concepción orteguiana subsiste la función puramente transmisora y reproductora del sistema de ideas o del proceso de racionalización del mundo humano y no humano. Es decir, la misión universitaria no deja de ser ilustración a la altura de su tiempo. Todo ello, aun siendo valioso y un suelo del que partir, no le imprime una tarea ética propia, pues no hay una pregunta fundamental por la "racionalidad de lo racionalizado", y por tanto, el cuestionamiento de los límites de las ideas vigentes en tanto que se revelen insuficientes o contradictorias para la marcha personal, social y natural.

7 "Por desgracia [sub. mío] no existe un puente que desde el análisis puramente empírico de la realidad efectuado con los instrumentos de la explicación causal conduzca a la confirmación o refuta- 
ción de la "validez" de cualquier juicio de valor" (Weber, 1985, p. 73).

8 He analizado esta cuestión al hilo de la encíclica Laudato si' en "Antropocentrismo y modernidad. Una crítica postilustrada" (Senent de Frutos, 2016).

9 En este contexto, señala el Papa Francisco (2015): "algunos rechazan con fuerza la idea de un Creador, o la consideran irrelevante, hasta el punto de relegar al ámbito de lo irracional la riqueza que las religiones pueden ofrecer para una ecología integral y para un desarrollo pleno de la humanidad. Otras veces se supone que constituyen una subcultura que simplemente debe ser tolerada" (no 62).

10 A modo de ejemplo, puede señalarse la acogida favorable de parte de la comunidad científica y de la comunidad internacional en cuanto a la contribución pública de la encíclica Laudato si (Camacho Laraña, 2016, pp. 60-61).

11 Congregación General 32, Decreto 4, n.2.

12 Horizonte sociocultural de donde surge la tradición judeocristiana que alumbró a la Iglesia y en donde se sitúa la Compañía de Jesús (Cf. Decreto 4 "Nuestra misión y la justicia" en Congregación General 34).

13 Como recuerda Josep Miralles, esta idea de justicia está en sintonía con el Concilio Vaticano II y en particular preanuanciada en el Sínodo Mundial de los Obispos de 1971, donde en su documento sobre "La justicia en el mundo" declaraban: "La acción a favor de la justicia y la participación en la transformación del mundo se nos presenta claramente como una dimensión constitutiva de la predicación del Evangelio, es decir, de la misión de la Iglesia para la redención del género humano y la liberación de toda situación opresiva".

14 Expresión que se viene usando sobre todo a partir de la Congregación General 32 y que se emplea igualmente en las posteriores.

15 Lo que en el documento "La promoción de la justicia en las universidades de la Compañía" elaborado por el Secretariado para la Justicia Social y Ecología (2014, p. 12) se denomina un "conjunto articulado de dimensiones de nuestra misión".

16 Cf. Secretariado para la Justicia Social y la Ecología (2013).

17 Son propuestas para ser debatidas en la Congregación General, presentadas por jesuitas o por la Congregación Provincial con carácter previo a la misma.
18 Secretariado del Apostolado Social de la Curia General de la Compañía de Jesús, 1999.

19 Subrayado mío.

20 La globalización ha cortado las líneas de fuga de la "planicie" de la tierra, ésta ya no es ilimitada e inconmensurable, es un lugar limitado donde todos ocupan una posición, donde a unos pocos les va "bien" en la vida, y a la mayor parte les va mal. En tiempos de Ignacio se abrían nuevos horizontes geográficos, hoy esos horizontes ya están recorridos, pero la historia del sufrimiento humano nos sigue desafiando como entonces (Ignacio de Loyola, 1997).

21 Accedemos a esta información gracias al portal web de la Congregación General 36, y a través de la entrevista a Juan Rafael Velasco, SJ, donde se anuncian los ejes de los dos decretos fruto de esta congregación que serán publicados a comienzos de 2017: https://www.youtube.com/watch?v=vwfKL8g9_BM

22 He tratado de plantear una hermenéutica histórica que responda a los desafíos socio-históricos actuales desde la tradición ignaciana en "Hacia una relectura de la matriz cultural ignaciana desde nuestras necesidades civilizatorias actuales" (2014); así como una aplicación de lo anterior en "Aspectos contextuales y metodológicos del diálogo de las espiritualidades con las disciplinas universitarias. Tradición ignaciana y jesuita como fuente de renovación de la formación universitaria" (2015).

23 Así por ejemplo, cabe destacar en el ámbito de educación promovida desde la Compañía, universitaria o de otros niveles, la formulación de un modo de proceder propio, el paradigma pedagógico ignaciano, que tiene su origen en los Ejercicios Espirituales de s. Ignacio, las Constituciones de la Compañía, y en su aplicación pedagógica en la $R a-$ tio Studiorum de la primera Compañía. Sobre esto puede verse Gil Coria y Labrador (2002).

24 Entre otros, Rambla, 2011, pp. 53-82; Romero Rodríguez, 2007, pp. 398-400 y Secretariado para la Justicia Social y la Ecología, 2011, p. 13.

25 El pionero a nivel general para la Compañía universal fue "Vivimos en un mundo roto. Reflexiones sobre la Ecología" (Secretariado del Apostolado Social, 1999), que recogía el estudio encargado por la Congregación Gene- ral 34. A este estudio siguió, al hilo de la Congregación General 35, "Sanar un mundo herido. Informe especial sobre Ecología" (Secretariado para la Justicia Social y la Ecología, 2011).

26 Destaco el número monográfico Una espiritualidad que nos reconcilia con la creación (Secretariado para la Justicia Social y la Ecología, 2013).

27 García (2014) indica: “¿Tiene la espiritualidad ignaciana alguna inspiración propia que poner al servicio del movimiento ecologista? Creemos que sí (...). Tal vez la raíz más honda de esa afirmación la tengamos en el comienzo y el final de los Ejercicios, en el Principio y Fundamento (PyF) y en la Contemplación para alcanzar amor (CaA): Las 'cosas' son más que cosas. Son creaturas y dones de Dios en los que Dios mismo habita y nos espera. El proceso de los Ejercicios queda enmarcado en una doble afirmación. La del PyF: que no sólo el hombre sino 'las otras cosas sobre la haz de la tierra,' todas ellas, son creadas por Dios, es decir provienen de su amor originante. $\mathrm{Y}$ la de la $\mathrm{CaA}$ : que toda realidad es lugar de encuentro con ese Dios que es y se manifiesta: a) dándonos las cosas y dándosenos en ellas; b) habitándolas; c) trabajando en ellas por nosotros; d) descendiendo a ellas".

28 En este sentido, señala Nicolás "hasta ahora no hemos hecho uso pleno de este 'extraordinario potencial' para el servicio 'universal'” (Profundidad, universalidad y ministerio intelectual). Así también, en 2014 en el documento del Secretariado para Justicia Social y la Ecología, se reconoce: "Queda mucho por hacer en la realización de la colaboración global posible en la educación universitaria de la Compañía" ( $p$. 34). Aunque hay que señalar que ese camino ya está en parte recorriéndose con la contribución de universidades jesuitas y centros de investigación a las Redes Globales de Advocacy Ignaciano (GIAN), articuladas en cinco ejes: ecología; el derecho a la educación para todas las personas; gobernanza de recursos naturales y minerales; migrantes y desplazados; y paz y derechos humanos (Secretariado para Justicia Social y la Ecología, 2013).

29 A esta cuestión dedicó la Revista de Fomento Social un monográfico titulado "El binomio identidad y misión ante los desafíos universitarios" (VV. AA, 2008) 


\section{BIBLIOGRAFÍA}

Álvarez de los Mozos, P. (2016). Defender al pobre y proteger la naturaleza. Revista de Fomento Social, 71, pp. 81-98.

Benedicto XVI (2011). Discurso al parlamento alemán. [En línea]. Disponible en http://www.vatican.va/holy_ father/benedict_xvi/speeches/2011/ september/documents/hf_benxvi_spe_20110922_reichstag-berlin_ sp.html"

Camacho Laraña, I. (2016). Laudato si': e clamor de la tierra y el clamor de los pobres. Una encíclica más que ecológica. Revista de Fomento Social, 71, pp. 69-79.

Francisco (2015). Carta encíclica Laudato si'. Sobre el cuidado de la casa común. Disponible en http://w2.vatican.va/ content/francesco/es/encyclicals/documents/papa-francesco_20150524_enciclica-laudato-si.htm

Gil Coria, E. y Labrador, C. (eds.). (2002). La pedagogía de los jesuitas, ayer y hoy. Madrid: Universidad Pontificia Comillas.

Guibert, J. M. (2014). Diccionario de liderazgo ignaciano. Bilbao: Mensajero.

Ignacio de Loyola (1997). Obras. Madrid: BAC.

Kolvenbach, P. H. (1989/2008). En el segundo centenario de la enseñanza jesuítica en los Estados Unidos de América. En Agúndez Agúndez, M. (ed.). Kolvenbach, P. H. Discursos Universitarios. Madrid: UNIJES, pp. 74-86.

Kolvenbach, P. H. (2000/2008). El servicio de la fe y la promoción de la justicia en la Educación Universitaria de la Compañía de Jesús en Estados Unidos. En Agúndez Agúndez, M. (ed.) Kolvenbach, P. H. Discursos Universitarios. Madrid: UNIJES, pp. 169-189.

Kolvenbach, P. H. (2001/2008). La universidad de la Compañía de Jesús a la luz de carisma ignaciano. En Agúndez Agúndez, M. (ed.). Kolvenbach, P. H. Discursos Universitarios. Madrid: UNIJES, pp. 190-209.

Kolvenbach, P. H. (2006/2008). Lección inaugural en la Universidad San Alberto Hurtado, Santiago de Chile, 1 mayo 2006. En Agúndez Agúndez, M. (ed.) Kolvenbach, P. H. Discursos Universitarios. Madrid: UNIJES, pp. 224-235.

López Casquete de Prado, M. (2016). Ecología y desarrollo humano integral. Revista de Fomento Social, 71, pp. 161-165.
Ortega y Gasset, J. (1957). La misión de la Universidad. En Obras completas (4a ed). Madrid: Revista de Occidente, pp. 313-353.

Rambla, J. M. (2011). Espiritualidad ignaciana y lucha por la justicia. En Alegre, X. González Faus, J. I., Rambla, J. M., Giménez, J., Juan, G. y Javier Vitoria, F. Mística y compromiso por la justicia. Barcelona: Cristianisme i Justícia.

Romero Rodríguez, J. J. (2007). Misión de una Universidad jesuita: retos y líneas de futuro. Revista de Fomento Social, 62, pp. 393-418.

Secretariado del Apostolado Social de la Curia General de la Compañía de Jesús (1999). Vivimos en un mundo roto. Reflexiones sobre la Ecología. Promotio Iustitiae, 70

Secretariado para la Justicia Social y la Ecología (2011). Informe especial sobre Ecología. Sanar un mundo herido. Grupo de Trabajo sobre Ecología. Promotio lustitiae, 106. Disponible en http://www.sjweb.info/documents/sjs/ pjnew/PJ106ESP.pdf

Secretariado para la Justicia Social y la Ecología (2011). Invitados a colaborar. Dimensión de la justicia y retos apostólicos hoy en la Compañía de Jesús, desde la perspectiva del Apostolado Social. Disponible en Promotio Iustitiae, 107. Disponible en http://www.sjweb.info/ sjs/documents/PJ_107_ESP.pdf

Secretariado para la Justicia Social y la Ecología (2013). Con pasión por la justicia medioambiental. Respuesta de la Compañía de Jesús al reto de "reconciliarnos con la creación". Promotio lustitiae, 110. Disponible en http://docplayer. es/23738539-Promotio-iustitiae-conpasion-por-la-justicia-medioambientalrespuestas-de-la-compania-al-reto-dereconciliarnos-con-la-creacion.html

Secretariado para la Justicia Social y la Ecología (2013). Reseaux internationaux de plaidoyer ignatien (GIAN). Promotio lustitiae, 110. Disponible en http:// www.sjweb.info/sjs/documents/ PJ 110 FRA.pdf

Secretariado para la Justicia Social y la Ecología (2013). Una espiritualidad que nos reconcilia con la creación. Promotio lustitiae, 111. Disponible en http://www. comboni.org/risorse/allegati/1041.pdf
Secretariado para la Justicia Social y la Ecología (2014). La promoción de la Justicia en las Universidades de la Compañía. Promotio lustitiae, 116. Disponible en http://www.unijes.net/?wpfb_dl=45

Secretariado para la Justicia Social (2006). Globalización y Marginación. Nuestra respuesta apostólica global. Disponible en http://www.sjweb.info/sjs/documents/ GlobMarg_ESP.pdf"

Senent de Frutos, J. A. (2009). Ecología y problemas medioambientales. Orientaciones para la praxis desde otro marco cultural. Revista de Fomento Social, 64, pp. 699-708.

Senent de Frutos, J. A. (2014). Hacia una relectura de la matriz cultural ignaciana desde nuestras necesidades civilizatorias actuales. Revista latinoamericana de teología, 93, pp. 247-270.

Senent de Frutos, J. A. (2015). Aspectos contextuales y metodológicos del diálogo de las espiritualidades con las disciplinas universitarias. Tradición ignaciana y jesuita como fuente de renovación de la formación universitaria. En FornetBetancourt, R. (ed.).Tradiciones de formación, espiritualidad y universidad. Hacia una transformación intercultural de la formación académica. Wissenschaftsverlag Mainz, pp. 93-105.

Senent de Frutos, J. A. (2016). Antropocentrismo y modernidad. Una crítica postilustrada. Revista de Fomento Social, 71, pp. 107-114.

Sínodo Mundial de los Obispos (1971). La justicia en el mundo. Disponible en http://www.confer.es/508/activos/texto/wcnfr_pdf_2566-U1bgv1kNzDHk$7 \mathrm{cr} 2 . \mathrm{pdf}$

Terrén, E. (1996). Las aulas desencantadas: Max Weber y la educación. Política y Sociedad, 21, pp. 133-148.

VV. AA. (2008). El binomio identidad y misión ante los desafíos universitarios. Revista de Fomento Social, 252.

Weber, M. (1985). El problema de la irracionalidad en las ciencias sociales. Madrid: Tecnos.

Weber, M. (2003). El político y el científico. Madrid: Alianza Editorial.

Weber, M. (2009). La "objetividad" del conocimiento científico en la ciencia social y en la política social. Madrid: Alianza Editorial. 


\section{Recursos de Internet}

Diálogo del Papa Francisco con la 36a Congregación General de la Compañía de Jesús. [En línea]. Disponible en http:// www.laciviltacattolica.it/articoli_download/extra/DIALOGOPAPA_ESP.pdf

García, J. A. (2014). Ecología y espiritualidad ignaciana. [En línea]. Disponible en http://www.ecojesuit.com/ecologia-yespiritualidad-ignaciana/6377/?lang=es

Remolina, G. (2012). Del "Big Bang" de las ciencias a la integración del saber. Reflexiones sobre la interdisplinariedad. Lección inaugural. Año académico 2012. Universidad Centroamericana. Managua, Nicaragua. [En línea]. Disponible en http://repositorio.uca.edu. ni/868/1/leccion_inaugural_2012.pdf

Vida y misión de la Compañía de Jesús en el Colegio Centro América. [En línea]. Dis- ponible en https://www.youtube.com/ watch?v=vwfKL8g9_BM

\section{Otras fuentes}

Compañía de Jesús. Congregación General 32. Madrid: Razón y Fe, 1975. Disponible en http://www.sjweb.info/sjs/documents/CG32_D2_esp.pdf

Compañía de Jesús. Congregación General 34. Bilbao: Mensajero, 1995. Disponible en http://www.sjweb.info/documents/ education/CG34_D17_SPA.pdf

Compañía de Jesús. Congregación General 35. Bilbao: Mensajero, 2008. Disponible en http://www.sjweb.info/35/documents/ Decretos.pdf

Eguiluz, J. (1995). Introducción al decreto sobre la Compañía y la vida universitaria. En Compañía de Jesús. Congregación General 34. Bilbao: Mensajero, pp. 339-343,
Miralles, J. (1995). Introducción a la Recomendación sobre Ecología al Padre General. En Compañía de Jesús. Congregación General 34. Bilbao: Mensajero, pp. 363-364.

Nicolás, A. Profundidad, universalidad y ministerio intelectual. Retos para la educación superior jesuita hoy. Comentarios para "Redes para la Educación Superior Jesuita: configurar un futuro para un mundo humano, justo y sostenible". Ciudad de México, 23 abril 2010. [En línea]. Disponible en http://www. sjweb.info/documents/ansj/100423_ Mexico_ESP.pdf

Nicolás, A. Sobre los jesuitas destinados al apostolado intelectual. Carta a toda la Compañía (Roma, 24 de mayo de 2014). 